\title{
DAS FELLOW-PROGRAMM FREIES WISSEN: OPEN SCIENCE SELBST PROBIERT
}

\author{
von Martina Trognitz
}

Zusammenfassung: Der Aufsatz gibt die persönlichen Erfahrungen der Autorin mit offener Wissenschaft während des Studiums und als Fellow des Fellow-Programms Freies Wissen ${ }^{1}$ wieder. Einleitend wird eine kurze Einführung zum Begriff „Open Science", offene Wissenschaft, sowie den damit zusammenhängenden Prinzipien gegeben, um anschließend über deren Anwendung in den Studiengängen Klassische Archäologie und Computerlinguistik zu reflektieren. Es folgt eine Vorstellung des Fellow-Programms und ein Überblick der Aktivitäten und die praktische Anwendung der Prinzipien anhand eines Projektes zur Erstellung einer interaktiven, offenen Online-Bibliografie während der achtmonatigen Laufzeit der Programmrunde 2018/2019.

Schlagwörter: offene Wissenschaft; Stipendium; Wikidata

\section{THE OPEN SCIENCE FELLOWS PROGRAM: PRACTICING OPEN SCIENCE}

Abstract: The paper presents the personal experience of the author with Open Science during her academic studies and as a fellow of the Fellow-Programm Freies Wissen. The concept "Open Science" is introduced along its accompanying principles. The author reflects upon the application of those principles during her studies of Classical Archaeology and Computational Linguistics. An introduction to the Fellow-Programme is also provided, along an overview of the author's activities and implementation of the principles during the eight months period of the programme 2018/2019, where a project to create an interactive, open, and online bibliography was pursued.

Keywords: Open Science; Fellowship; Wikidata

DOI: https://doi.org/10.31263/voebm.v72i2.3033

(C) Martina Trognitz

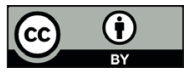

Dieses Werk ist lizenziert unter einer

Creative-Commons-Lizenz Namensnennung 4.0 International 


\section{Inhalt}

1. Open Science

2. Open Science im Studium?

3. Das Fellow-Programm Freies Wissen

4. Meine Erfahrungen im Programm

5. Fazit

\section{Open Science}

Der Begriff „Open Science“, offene Wissenschaft, ist mir als solcher erst 2017 bewusst begegnet. Damals hat das Open Innovation in Science Center der Ludwig Boltzmann Gesellschaft ${ }^{2}$ einen Workshop mit dem Titel „Open Science Workshop - What's in it for me?" organisiert. Neben einer Fülle an vorgestellten Tools ${ }^{3}$ und Informationsquellen wurde natürlich auch eine allgemeine Einführung zum Begriff „open“, bzw. „offen“ anhand der Open Definition ${ }^{4}$ gegeben.

Anhand weniger Punkte, jedoch präzise, wird darin definiert, was mit „offen“ in Bezug auf Wissen, Daten und andere Inhalte gemeint ist: „Wissen ist offen, wenn jedeR darauffrei zugreifen, es nutzen, verändern und teilen kanneingeschränkt höchstens durch Maßnahmen, die Ursprung und Offenheit des Wissens bewahren."

Was „Open Science“ selbst bedeutet und ermöglichen kann, wird sehr eindrucksvoll von Michael Nielsen in einem TEDx-Vortrag ${ }^{6}$ beschrieben. Nielsen argumentiert, dass die Digitalisierung und vor allem die Vernetzung über das Internet genutzt werden können, um neue Werkzeuge und Möglichkeiten zu schaffen, die eine Bearbeitung höchst komplexer Fragestellungen zulassen. Als anschauliches Beispiel erwähnt er das Polymath-Projekt, ${ }^{7}$ das durch den Mathematiker Tim Gowers ${ }^{8}$ angestoßen wurde. Mit Hilfe eines Blogbeitrages, ${ }^{9}$ in dem Gowers die Fragestellung sowie seine bisherigen Gedankengänge und Lösungsansätze zu einem mathematischen Problem vorstellte und gleichzeitig eine offene Einladung zur Beteiligung aussprach, wurde das Problem innerhalb kürzester Zeit gelöst und publiziert. ${ }^{10}$ Nielsen betont in seinem Vortrag auch, dass Open Science ein Umdenken erfordert und aktiv von Wissenschaftlerinnen und Wissenschaftlern sowie der Öffentlichkeit betrieben und eingefordert werden muss. Aufgrund der Beschleunigung der Forschung, der Erweiterung der zu bearbeitenden Fragestellungen und damit einhergehenden Erweiterung des Wissens, ist offene Wissenschaft jedoch ein lohnenswertes Unterfangen mit ungeahnten Möglichkeiten. 
So wie der wissenschaftliche Prozess selbst verschiedene Aspekte und Aktivitäten beinhaltet, können für offene Wissenschaft ebenfalls mehrere Bestandteile unterschieden werden, die wiederum jeweils offen gestaltet werden können. Stefan Kasberger und Christopher Kittel von openscienceASAP ${ }^{11}$ unterscheiden hierbei sechs Bestandteile oder Prinzipien: Open Methodology, Open Source, Open Data, Open Access, Open Peer Review und Open Educational Resources. Wikimedia Deutschland ${ }^{12}$ zählt außerdem noch Citizen Science als Komponente dazu. In der Grafik in Abbildung 1 sind diese Prinzipien zusammengefasst.

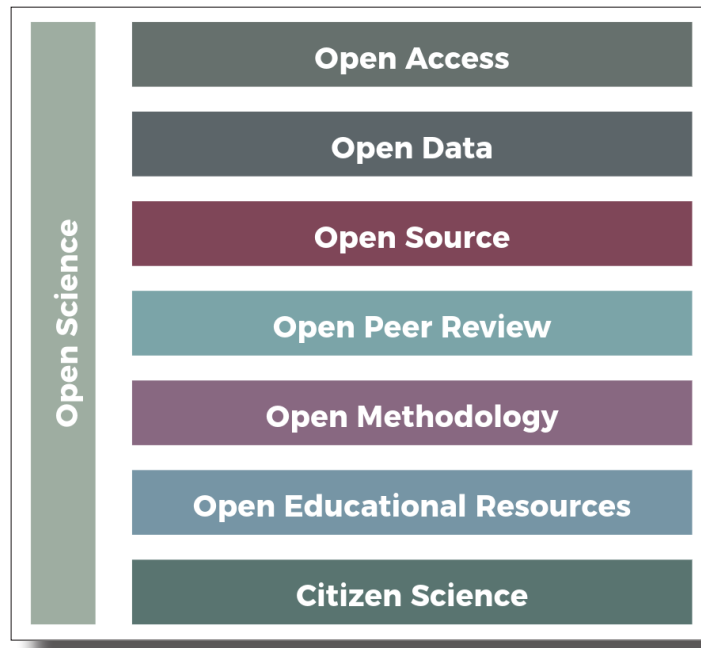

Abb. 1: Die sieben Prinzipien oder Teilaspekte von Open Science. [Markus Büsges, leomaria designbüro - CC BY-SA 4.0 (https://creativecommons.org/licenses/by-sa/4.0)]

Diese sieben Prinzipien entsprechen Aktivitäten des wissenschaftlichen Arbeitens. Mit Open Methodology ist die Offenlegung und offene Dokumentation der angewandten Methoden und Prozesse gemeint. Open Source und Open Data beschreiben die Verwendung frei nutzbarer Soft- und Hardware, respektive Daten, sowie deren Öffnung bei deren Erstellung. Auch Open Access bezieht sich auf freie Zugänglichkeit, hier in Bezug auf Publikationen. Im Zusammenhang mit der Qualitätssicherung von Publikationen oder Daten steht Open Peer Review, das ein nachvollziehbares Peer-Review-Verfahren meint. Die Bereitstellung und Verwendung von freien Bildungs- und Lehrmaterialien wird unter Open Educational Resources zusammengefasst. Citizen Science wiederum ist die Einbeziehung der allgemeinen Öffentlichkeit in den Forschungsprozess. 


\section{Open Science im Studium?}

Ein eigenes Thema war Open Science während meines Studiums der Computerlinguistik und Klassischen Archäologie an der Universität Heidelberg nicht. Auch kann ich mich nicht daran erinnern, dem Begriff in einer Vorlesung oder Übung begegnet zu sein. Dies könnte zum Einen daran liegen, dass der Lehrkörper selbst noch nicht viel Erfahrung mit oder kein Interesse an offener Wissenschaft hat. Ein anderer Grund könnte damit zusammenhängen, dass meine Studienzeit bald zehn Jahre zurückliegt und die Open-Science-Bewegung erst in jüngerer Zeit an Fahrt aufgenommen hat, wie die mit Scholia erstellte Abbildung 2 zeigt. Das Tool Scholia ${ }^{13}$ wurde für die Visualisierung wissenschaftlicher Publikationen konzipiert und verwendet dazu Informationen aus Wikidata. Da nicht alle wissenschaftlichen Publikationen in Wikidata eingetragen sind, gibt die Grafik jedoch nicht das vollständige Bild wieder. Zu betonen ist, dass die Publikationen von 1979, 1985 und 1998 sich ebenfalls schon mit Open Science in dem in diesem Heft thematisierten Sinn befassen.

\subsection{Unterschiede in den Fächern}

Was mir in der nachträglichen Reflexion über die vermittelten Werte und Arbeitsweisen während des Studiums aufgefallen ist, sind die verschiedenen disziplinspezifischen Praktiken, die auch einen unterschiedlichen Grad der Öffnung aufweisen.

In der Archäologie drängt sich das Bild des einsamen Wissenschaftlers im Elfenbeinturm auf, da man meist für sich allein in der Bibliothek arbeitete. Denn Referate wurden jeweils von einer Person gehalten und die wichtigsten Quellen sind gedruckte Monographien und Aufsätze, die kaum online verfügbar waren (oder sind). Für das Bildmaterial für Vortragsfolien stand eine institutseigene interne Bilddatenbank zur Verfügung. Andere Datenbanken gab es auch, jedoch waren diese ebenfalls zugriffsbeschränkt. Immerhin gibt es in diesem Bereich mittlerweile gute offene Alternativen, wie Arachne ${ }^{14}$ oder Wikimedia Commons. ${ }^{15}$

Anders gestaltete sich die Computerlinguistik. Referate wurden meist in Kleingruppen gehalten, Softwarepraktika in kleinen Teams durchgeführt und auch Übungszettel durften explizit in Gruppen bearbeitet werden. Die Materialien für die Vorträge waren zum größten Teil online verfügbar, so wie auch die Folien und Skripte aller Veranstaltungen. Insgesamt wurde hier also eine offenere Arbeitsweise vorgelebt und vermittelt. 


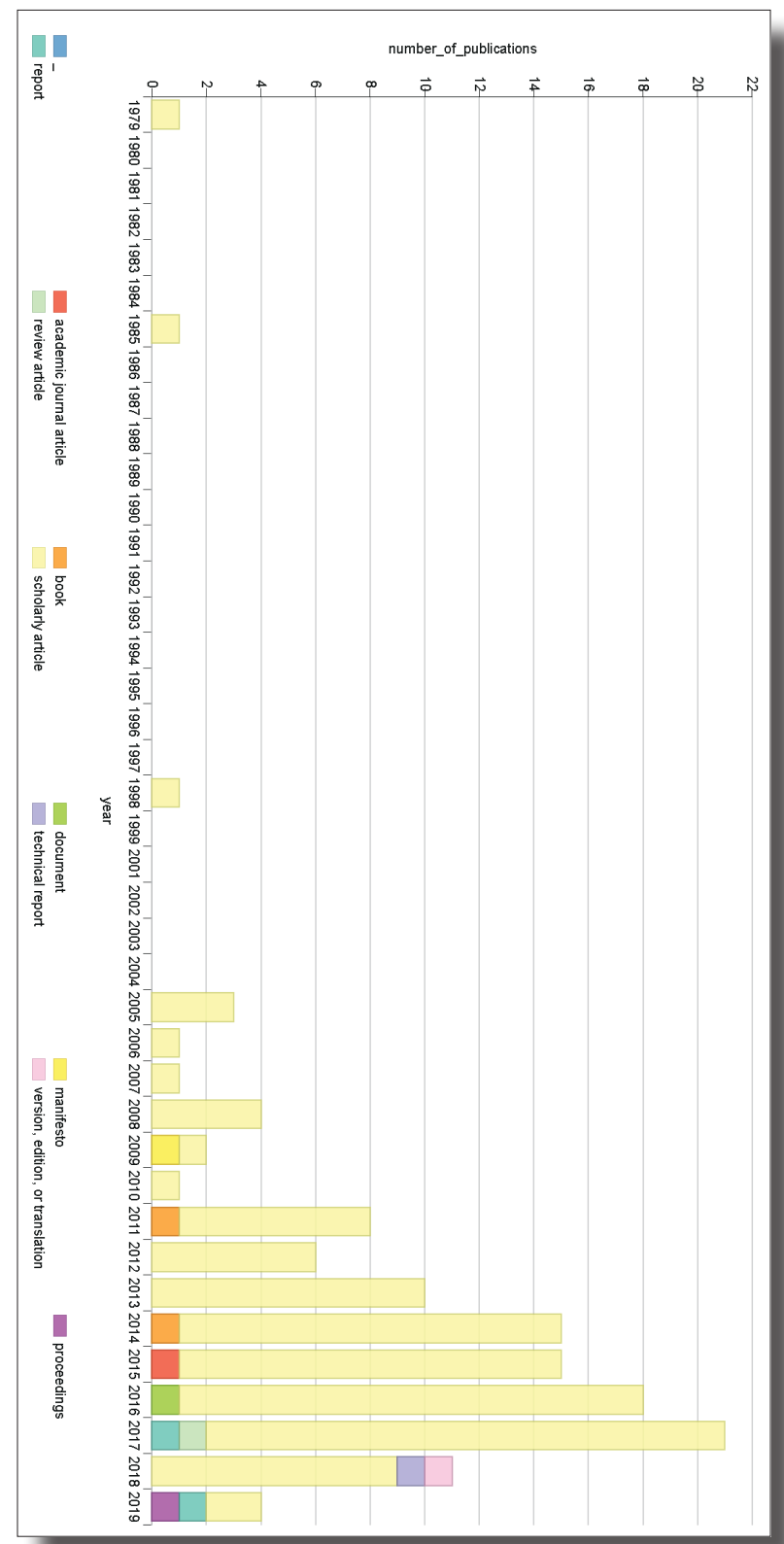

Abb. 2: Entwicklung der Anzahl an Publikationen zum Thema "Open Science" seit 1979. [Grafik erstellt von der Autorin mit Scholia: https://tools.wmflabs.org/scholia/topic/ Q309823 (09.08.2019)] 
Meine Orientierung hin zur Bearbeitung archäologischer Fragestellungen mit rechnergestützten Methoden und zu den Digital Humanities im Allgemeinen, bestärkt meine Vermutung, dass mit dem Einsatz digitaler Methoden und Werkzeuge auch die Offenheit gefördert wird. In den Digital Humanities wird vor allem Programmcode frei zur Nachnutzung verfügbar gestellt und aufgrund des Zusammentreffens verschiedener Spezialisierungen ist Teamarbeit nichts Ungewöhnliches. Publikationen sind zwar nicht alle als Open Access verfügbar, jedoch verhältnismäßig stärker vertreten als etwa klassisch archäologische Publikationen ohne oder mit einem sehr geringen Anteil an digitalen Methoden. Als Indiz dafür kann ein Vergleich von Publikationslisten dienen. In der Liste, die von der European Association for Digital Humanities ${ }^{16}$ zusammengestellt wurde, sind zwei Drittel, also etwa $66 \%$, der gelisteten Publikationen entweder Open Access Journale oder andere offen verfügbare Materialien. Wenn man die ersten 50 Einträge der Liste von archäologischen Zeitschriften und Reihen des American Journal of Archaeology ${ }^{17}$ auf Konformität mit Open Access prüft, reduziert sich der Anteil auf ein Fünftel, also 20\%. Hierbei sei aber erwähnt, dass bei Einbeziehung von Retrodigitalisaten nach Ablauf der Regelschutzfrist und einer sogenannten „Moving Paywall“ der Anteil auf ca $50 \%$ steigt.

Dass es Unterschiede in den verschiedenen Fachrichtungen bei der Anwendung von Prinzipien der offenen Wissenschaft gibt, geht auch aus einem Beitrag von José van Dijck zur Open-Science-Agenda der Niederländischen Königlichen Akademie der Wissenschaften hervor, in dem es zum Schluss heißt, dass Open Science erfolgreich sein kann, wenn Unterschiede zwischen den Disziplinen zugelassen werden. ${ }^{18}$ Dem wird auf der nationalen Plattform für Open Science in den Niederlanden ebenfalls mit einer kurzen Erwähnung Rechnung getragen, in der betont wird, dass die Etablierung offener Wissenschaft von der Fachdisziplin abhängig ist und daher fachspezifische Übereinkommen zur Förderung von offener Wissenschaft notwendig sind. ${ }^{19}$

Veröffentlichungen darüber, worin genau die Unterschiede liegen und Studien, die belegen wie offen ein Fach ist, sind mir nicht bekannt. Die Durchführung einer interdisziplinären Studie zu diesem Thema wäre also angebracht. Dies darf man als Aufruf zu einem kollaborativen Projekt verstehen.

\section{Das Fellow-Programm Freies Wissen}

Um der Open-Science-Bewegung zu einem Aufschwung zu verhelfen, wurde 2016 das Fellow-Programm Freies Wissen ${ }^{20}$ ins Leben gerufen. Es han- 
delt sich um ein Förderprogramm der Wikimedia Deutschland, ${ }^{21}$ dem Stifterverband ${ }^{22}$ und der VolkswagenStiftung, ${ }^{23}$ das dieses Jahr in die vierte Runde gestartet ist und dessen wichtigstes Anliegen die stufenweise Öffnung der Wissenschaft ist, wobei dabei die kollaborative Arbeitsweise auf Wikipedia als Vorbild dient. ${ }^{24}$ Gefördert werden Projekte aus allen Fachdisziplinen, welche vom Assistenten bis hin zur Juniorprofessorin eingereicht werden können.

Über eine Zeit von acht Monaten gibt es ein Qualifizierungsprogramm mit Workshops an drei Präsenzterminen, sowie Webinare, die über die Prinzipien von Open Science informieren und zum Nachdenken anregen. Jeder Fellow bekommt für die gesamte Laufzeit eine Mentorin oder Mentor zur Seite gestellt, mit denen auch ein regulärer Austausch erfolgt. Das Qualifizierungsprogramm und das Mentoring dienen dabei nicht nur dem Fortkommen des Projektes und der Vermittlung der Grundlagen von offener Wissenschaft, sondern auch der erhöhten Sichtbarkeit und Vernetzung der Fellows und deren Projekte. Eine finanzielle Unterstützung wird als Bonus gewährt.

Ein wesentlicher Aspekt des Fellow-Programms ist die Wirkung der Fellows als Multiplikatoren. Denn letztendlich kann Open Science nur durch aktive Partizipation und kontinuierliche Information, sowohl innerhalb der Forschung als auch in der Gesellschaft allgemein, gestärkt werden. Wie eingangs erwähnt, hat dies Michael Nielsen ebenfalls schon festgestellt.

\section{Meine Erfahrungen im Programm}

An dieser Stelle möchte ich von meinen Erfahrungen berichten, die ich während des Fellow-Programms Freies Wissen 2018/2019 gemacht habe. Im Rahmen meines Vorhabens habe ich mir dabei vorgenommen die verschiedenen Prinzipien offener Wissenschaft möglichst umfassend anzuwenden.

\subsection{Bewerbungsprozess}

Der Bewerbungsprozess war mit dem Schreiben eines kurzen Antrags vergleichbar. Neben einer Projektskizze waren Angaben zu Erfahrungen und Umgang mit offener Wissenschaft erforderlich. Die Beweggründe zur Teilnahme an dem Projekt sollten ebenso wie die erhoffte Wirkung des Vorhabens skizziert werden

Während der Bewerbung trat für mich schon der erste Lerneffekt ein, denn die Frage nach geplanten oder möglichen Beiträgen zu den Projek- 
ten der Wikimedia erforderte eine eingehendere Beschäftigung mit den Wikimedia-Projekten. ${ }^{25}$ Schnell stellte ich fest, dass es weit mehr als nur Wikipedia ${ }^{26}$ als freies Nachschlagewerk, Wikimedia Commons ${ }^{27}$ als zentrale freie Medienplattform und Wiktionary ${ }^{28}$ als offenes Wörterbuch gibt. Weitere Projekte von Wikimedia sind unter anderem das Artenverzeichnis Wikispecies, ${ }^{29}$ Wikiversity ${ }^{30}$ als eine Plattform für Open Educational Resources, das Portal Wikivoyage ${ }^{31}$ mit Reiseinformationen aus aller Welt, sowie Wikidata ${ }^{32}$ für strukturierte Daten. Wikidata sollte letztendlich auch das wichtigste Mittel für mein Projektvorhaben werden.

Mein Projekt hatte zum Ziel, die Referenzen aus einer gedruckten Bibliografie zum Thema ägäische Glyptik in Wikidata einzutragen. Mit einer eigens erstellten Webapplikation können die Referenzen anschließend angezeigt und nach Thema, Ort oder sogar Objekt durchsucht und gruppiert werden. Die Idee dabei ist, dass durch die Verwendung eines offenen, für jeden einseh- und bearbeitbaren Datenspeichers für die bibliografischen Informationen, auch Neuerscheinungen und bisher unbekannte Titel von allen ergänzt werden können, auch nach Ablauf des Projektes. Der Bewerbungstext, sowie die ausführliche Projektdokumentation sind online auf Wikiversity ${ }^{33}$ abrufbar.

\subsection{Die ersten vier Monate}

Der offizielle Start der Programmrunde 2018/2019 wurde mit einer Auftaktveranstaltung ${ }^{34}$ im September 2018 in Berlin eingeläutet. Hier hatte man die Gelegenheit zunächst die anderen Fellows, das Mentorenteam und vor allem die persönliche Mentorin bzw. den persönlichen Mentor kennen zu lernen. Das Schöne an dem breiten Zielpublikum des Programms ist, dass eine große Vielfalt an Fächern vertreten ist, die mit ihren Projekten nicht nur die Forschung direkt, sondern auch die Lehre und das Publikationswesen betreffen. In Abbildung 3 sind die vertretenen Fachdisziplinen nach Programmjahr dargestellt.

Während des Auftaktwochenendes wurden in Kleingruppen zusammen mit den Mentorinnen und Mentoren Roadmaps erstellt, die als Grundlage für die weitere Durchführung der Projekte dienen sollten. Wir diskutierten die Möglichkeiten, um die Projekte und deren Fortgang, ganz dem Prinzip Open Methodology entsprechend, öffentlich zu dokumentieren. Ich entschied mich letztendlich dafür, vor allem die oben erwähnte Dokumentation auf Wikiversity anzulegen und fortzuführen. Weitere beliebte Werkzeuge der Fellows für diesen Zweck waren Blogbeiträge, Tweets und Repositorien auf GitHub. ${ }^{35}$ Aktivitäten, die direkt das Programm betrafen, 
wurden kollaborativ auf den Seiten des Fellow-Programms auf Wikiversity hinterlegt, wie beispielsweise besuchte oder selbst organisierte Veranstaltungen oder auch inhaltliche Notizen aus den Qualifizierungsangeboten.

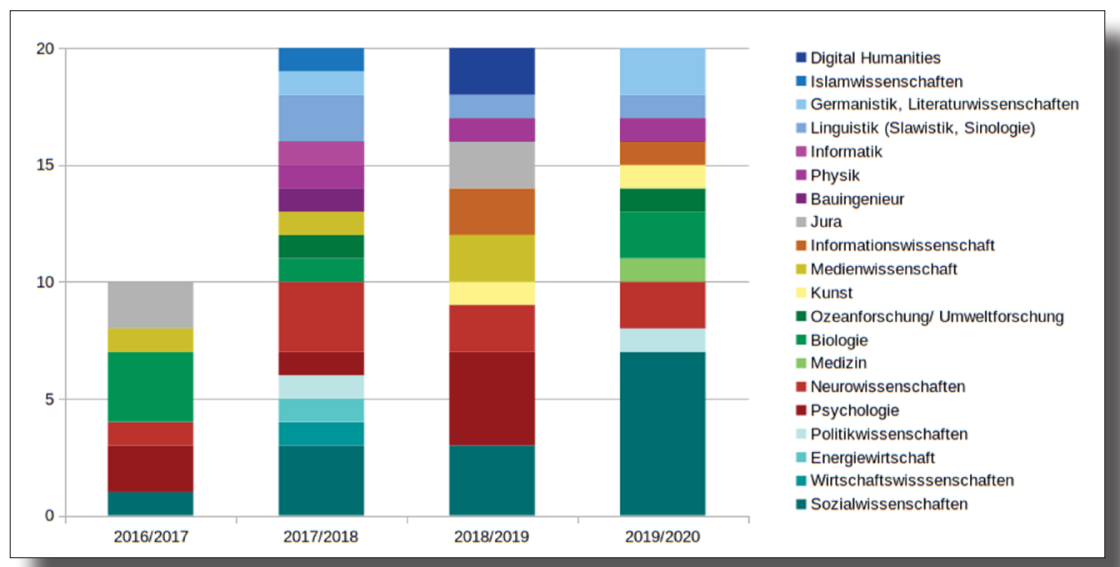

Abb. 3: Vertretene Fachdisziplinen in den vier Programmrunden des Fellow-Programms Freies Wissen. [Martina Trognitz - CC BY 4.0 (https://creativecommons.org/licenses/by/4.0)]

Inhalte auf Wikimedia-Seiten werden in einem eigenen Online-Texteditor unter Verwendung der sogenannten Wikisyntax ${ }^{36}$ eingegeben. Zunächst scheint diese nur sehr grundlegende Formatierungsmöglichkeiten zu bieten, wie verschiedene Überschriftsebenen, Kursiv- und Fettschreibung, Einfügen von Links usw. Mit der weiteren Auseinandersetzung stößt man jedoch schnell auf verschiedene über MediaWiki zur Verfügung gestellte Erweiterungen, ${ }^{37}$ wie beispielsweise EasyTimeline. ${ }^{38}$ Mit Hilfe dieser Erweiterungen können umfängliche Grafiken, Diagramme oder Bildergalerien erstellt werden. Die Zeitleiste ${ }^{39}$ auf meiner Projektbeschreibungsseite ist ein Beispiel für die sich eröffnenden Möglichkeiten.

Das Ende der ersten Halbzeit markierte ein Zwischenbericht, ${ }^{40}$ in dem der Zwischenstand im Projektverlauf abgefragt wurde und vor allem die Aktivitäten im Zusammenhang mit der Vernetzung und Kommunikation im Rahmen des Programms, sowie Aktivitäten und Initiativen mit Bezug zu offener Wissenschaft eine wichtige Rolle spielten.

\subsection{Die letzten vier Monate und Abschluss}

Mit zunehmendem Fortschritt meines Projektes wurden die Prinzipien Open Data und Open Source immer relevanter. Für die Speicherung der 
einzelnen Bestandteile der Literaturhinweise, wie Autorlnnenschaft, Titel, Erscheinungsort und Schlagworte, ist Wikidata das ideale System, um diese Informationen offen zugänglich zu speichern. Wichtig dabei ist ebenfalls, dass die Daten von allen bearbeitet und ergänzt werden können. Denn dies ist ganz im Sinne einer offenen und lebendigen Bibliografie, die jederzeit durch weitere Literaturhinweise ergänzt werden kann.

Da für die Dokumentation der einzelnen Importschritte die Projektseite auf Wikiversity nicht mehr der geeignete Platz war, wurde auf Wikidata eine Dataset-Imports-Seite ${ }^{41}$ angelegt. Sie dient als Einstiegspunkt zu den importierten Datensätzen und als Dokumentationsplattform der einzelnen Importschritte. Meine Aktivität auf Wikidata blieb nicht unbemerkt, was sich darin bemerkbar machte, dass andere Nutzer sowie verschiedene Bots (Skripte, die selbsttätig vorgegebenen Aufgaben verrichten) die von mir angelegten Einträge ergänzten und mit anderen verknüpften.

Für die Veröffentlichung des Quellcodes der entwickelten Applikation zur Anzeige der Informationen aus Wikidata war dann die Einrichtung eines Repositoriums auf GitHub die beste Option. ${ }^{42}$ Bei der Entwicklung der Anwendung, konnte ich selbst von anderen Open-Source-Projekten, wie ItemsJS, ${ }^{43}$ wcite $^{44}$ und Citation.js ${ }^{45}$ profitieren. Erste Kollaborationen haben sich über die Verwendung des offenen Software-Repositoriums ebenfalls ergeben.

Das Ende des Programms markierte die Abschlussveranstaltung ${ }^{46}$ in Berlin, auf der die Fellows mit Kurzpräsentationen von ihren persönlichen Erfahrungen der vergangenen acht Monate erzählten. Auch ein Abschlussbericht $t^{47}$ musste eingereicht werden, der sich zum Teil mit den Fragen des Zwischenberichtes deckte und zusätzlich Feedback zum Programm selbst abfragte.

\subsection{Nach dem Programm}

Die acht Monate waren schnell verflogen und für das Projektvorhaben insgesamt etwas kurz, doch das Ziel, Open Science am eigenen Leib auszuprobieren, wurde erreicht. Ich konnte viel lernen, Kontakte knüpfen und kann über den Alumni-Verteiler auch zukünftig in Kontakt mit den ehemaligen und zukünftigen Fellows bleiben.

Die Prinzipien offener Wissenschaft werde ich in der fortführenden Arbeit am Projekt weiterhin anwenden, sowie auf meine übrigen Tätigkeiten ausweiten. Insbesondere möchte ich die Prinzipien Open Access, Open Peer Review und Open Educational Resources noch anwenden, da ich im Rahmen der Laufzeit dazu keine Gelegenheit hatte. Das bedeutet konkret, 
dass die geplanten Publikationen über die Projektergebnisse in Open Access publiziert werden und anhand der gesammelten Erkenntnisse ein frei zugängliches Tutorial über die Vorbereitung und Bereinigung bibliografischer Informationen erstellt wird. Zu dem Thema Open Peer Review ist noch eine Einarbeitung erforderlich, doch entsprechende Unterlagen ${ }^{48}$ dafür sind auf dem Portal FOSTER ${ }^{49}$ zu finden, wie auch Informationen zu weiteren Fragen rund um Open Science.

\section{Fazit}

Die Teilnahme an dem Fellow-Programm Freies Wissen war lehrreich, sehr nützlich und ist nur zu empfehlen. Ich konnte zudem einen Einblick in andere Fachbereiche und deren Umgang mit Open Science erhalten. Die Beschäftigung mit offener Wissenschaft hat dazu angeregt über die eigene Arbeitsweise zu reflektieren. Dabei konnte ich feststellen, dass es Phasen in der wissenschaftlichen Tätigkeit gibt, die sich nicht ganz so leicht öffnen lassen, vor allem wenn man selbst noch mitten im Denkprozess steckt und noch nicht weiß wo es hingeht.

Ein Vorteil, der gleichzeitig auch ein Nachteil von Open Science ist, ist die Dokumentation des Prozesses und der Daten. Sie ist zeitaufwändig, aber der persönliche Nutzen zeigt sich sobald man nach einer längeren Pause die Arbeit an einem Projekt wieder aufnimmt und dank der Dokumentation den Faden schnell wieder aufnehmen kann.

Den im ersten Teil vorgestellten sieben Prinzipien würde ich noch „Dissemination" hinzufügen. Denn die Verbreitung des Wissens und die aktive Kommunikation darüber führt erst dazu, dass eine offene Wissenschaft im Miteinander stattfinden kann.

Zukünftig werde ich mich selbst immer fragen „Was geht offener?". Oder wie Michael Nielsen es so schön formuliert hat: „What are you doing to work more openly?"

Mag. ${ }^{a}$ Martina Trognitz ORCID: https://orcid.org/0000-0003-0485-6861 Österreichische Akademie der Wissenschaften, Austrian Centre for Digital Humanities (ACDH) E-Mail: martina.trognitz@oeaw.ac.at 
1 https://de.wikiversity.org/wiki/Wikiversity:Fellow-Programm_Freies_ Wissen (07.08.2019)

2 Ludwig Boltzmann Gesellschaft, Open Innovation in Science Center: https://ois.lbg.ac.at/

3 Bianca Kramer - Jeroen Bosman, 400+ Tools and innovations in scholarly communication <http://bit.ly/innoscholcomm-list> (23.05.2019)

4 Open Knowledge Foundation, Open Definiton 2.1 (2015) <http:// opendefinition.org/od/2.1/en/> (23.05.2019)

5 Open Knowledge Foundation, Offen-Definiton 2.1, Zusammenfassung (2015) <http://opendefinition.org/od/2.1/de/> (23.05.2019)

6 Michael Nielsen, Open Science, Vortrag bei TEDxWaterloo 2011 <http://michaelnielsen.org/blog/open-science-2/> (23.05.2019)

7 Polymath Blog: https://polymathprojects.org/

8 Tim Gowers, Is massively collaborative mathematics possible? (2009) <https://gowers.wordpress.com/2009/01/27/is-massively-collaborative-mathematics-possible/> (23.05.2019)

9 Tim Gowers, A combinatorial approach to density Hales-Jewett (2009) <https://gowers.wordpress.com/2009/02/01/a-combinatorial-approach-to-density-hales-jewett/> (23.05.2019)

10 D.H.J. Polymath, Density Hales-Jewett and Moser numbers (2010) <https://arxiv.org/pdf/1002.0374.pdf> (23.05.2019)

11 openscienceASAP, Was ist Open Science? <http://openscienceasap. org/open-science/> (23.05.2019)

12 Christopher Schwarzkopf (Wikimedia Deutschland), Warum Open Science? <https://de.wikiversity.org/wiki/Wikiversity:Fellow-Programm_ Freies_Wissen\%C2\%B4/Open_Science> (23.05.2019)

13 Scholia: https://tools.wmflabs.org/scholia/

14 Arachne, Version 3: https://arachne.uni-koeln.de/; Arachne Version 4: https://arachne.dainst.org/

15 Wikimedia Commons: https://commons.wikimedia.org/

16 EADH, Publications <http://eadh.org/publications/all> (23.05.2019)

17 American Journal of Archaeology, AJA Abbreviations, Journals and Book Series <https://www.ajaonline.org/submissions/journals-series> (23.05.2019)

18 José van Dijck, Open Science: Major Differences Between Disciplines <https://www.knaw.nl/en/topics/openscience/blog-jose-van-dijck-onopen-science> (23.05.2019)

19 National Platform Open Science, Discipline-specific agreements on the development of research support (2017) <https://www.openscience. $\mathrm{nl} / \mathrm{en} /$ themes/encouraging-and-supporting-open-science/discipline-specific-agreements-on-the-development-of> (23.05.2019) 
20 Wikimedia Deutschland, Fellow-Programm Freies Wissen auf Wikiversity < https://de.wikiversity.org/wiki/Wikiversity:Fellow-Programm_ Freies_Wissen> (23.05.2019)

21 Wikimedia Deutschland: https://wikimedia.de/wiki/Hauptseite

22 Stifterverband: https://www.stifterverband.org/freies-wissen

23 VolkswagenStiftung: https://www.volkswagenstiftung.de/

24 Christopher Schwarzkopf (Wikimedia Deutschland), Programm (2017-2019) <https://de.wikiversity.org/wiki/Wikiversity:Fellow-Programm_Freies_Wissen/Programm> (23.05.2019)

25 Wikimedia: https://www.wikimedia.org/

26 Wikipedia: https://www.wikipedia.org/

27 Wikimedia Commons: https://commons.wikimedia.org/

28 Wiktionary: https://www.wiktionary.org/

29 Wikispecies: https://species.wikimedia.org/

30 Wikiversity: https://www.wikiversity.org/

31 Wikivoyage: https://www.wikivoyage.org/

32 Wikidata: https://www.wikidata.org/

33 Martina Trognitz, A Linked and Open Bibliography for Aegean Glyptic in the Bronze Age (2018-2019) <https://de.wikiversity.org/wiki/Wikiversity:Fellow-Programm_Freies_Wissen/Einreichungen/A_Linked_ and_Open_Bibliography_for_Aegean_Glyptic_in_the_Bronze_Age> (23.05.2019)

34 Wikimedia Deutschland, Auftaktveranstaltung Programmjahr 2018/2019 <https://de.wikiversity.org/w/index.php?title=Wikiversity:Fellow-Programm_Freies_Wissen/Veranstaltungen/Auftaktveranstaltung_2018_2019> (23.05.2019)

35 Einen Teil der Projekte findet man auf: https://github.com/FellowsFreiesWissen

36 Wikisyntax: https://de.wikipedia.org/wiki/Hilfe:Wikisyntax

37 MediaWiki Extensions: https://www.mediawiki.org/wiki/Manual:Extensions

38 EasyTimeline: https://www.mediawiki.org/wiki/Extension:EasyTimeline

39 Martina Trognitz, A Linked and Open Bibliography for Aegean Glyptic in the Bronze Age - Zeitleiste (2018-2019) < https://de.wikiversity.org/ wiki/Wikiversity:Fellow-Programm_Freies_Wissen/Einreichungen/A_ Linked_and_Open_Bibliography_for_Aegean_Glyptic_in_the_Bronze_ Age\#Zeitleiste_/_Timeline> (23.05.2019)

40 Martina Trognitz, A Linked and Open Bibliography for Aegean Glyptic in the Bronze Age: Zwischenbericht (2019) < https://de.wikiversity.org/ wiki/Wikiversity:Fellow-Programm_Freies_Wissen/Einreichungen/A_ 
Linked_and_Open_Bibliography_for_Aegean_Glyptic_in_the_Bronze_ Age/Zwischenbericht> (23.05.2019)

41 Dataset-Imports-Seite auf Wikidata: https://www.wikidata.org/ wiki/Wikidata:Dataset_Imports/A_Bibliography_for_Aegean_Glyptic_in_the_Bronze_Age_(1991),_compiled_by_John_G._Younger (07.08.2019)

42 GitHub-Repositiorium zum Projekt: https://github.com/bellerophons-pegasus/lobib

43 ItemsJS: https://github.com/itemsapi/itemsjs

44 wcite: http://wikicite.org/wcite/

45 Citation.js: https://citation.js.org/

46 Wikimedia Deutschland, Abschlussveranstaltung Programmjahr 2018/2019 <https://de.wikiversity.org/wiki/Wikiversity:Fellow-Programm_Freies_Wissen/Veranstaltungen/Abschlussveranstaltung_2019> (15.06.2019)

47 Martina Trognitz, A Linked and Open Bibliography for Aegean Glyptic in the Bronze Age: Abschlussbericht (2019) <https://de.wikiversity.org/wiki/Wikiversity:Fellow-Programm_Freies_Wissen/Einreichungen/A_Linked_and_Open_Bibliography_for_Aegean_Glyptic_in_the_ Bronze_Age/Abschlussbericht> (23.05.2019)

48 FOSTER, Open Peer Review <https://www.fosteropenscience.eu/ node/2333> (15.06.2019)

49 FOSTER: https://www.fosteropenscience.eu/ 